\title{
Electron field emission from composite electrodes of carbon nanotubes-boron-doped diamond and carbon felts
}

\author{
J. Mauricio Rosolen, ${ }^{\text {a) }}$ Simone Tronto, Marcel S. Marchesin, Erica C. Almeida, ${ }^{\text {b) }}$ \\ Neidenei G. Ferreira, ${ }^{\text {b) }}$ C. H. Patrick Poá, ${ }^{\text {c) }}$ and S. Ravi P. Silva ${ }^{\text {d) }}$ \\ Nano-Electronics Centre, ATI, University of Surrey, Guildford GU2 7XH, \\ United Kingdom and Dep. de Química, FFCLRP, Universidade de São Paulo, SP-14040-930 Brazil
}

(Received 31 October 2005; accepted 27 January 2006; published online 23 February 2006)

\begin{abstract}
The electron field emission of carbon nanotube (CNT)/boron-doped diamond (BDD)/carbon felt electrodes (CNT/BDD/felt) have been investigated. The composite electrode was initially prepared with the growth of BDD on carbon felt and the subsequent growth of CNT by chemical decomposition of methanol. The composite electrodes were characterised using scanning electron microscopy and transmission electron microscopy. For the CNT/BDD/felt samples, the electron field

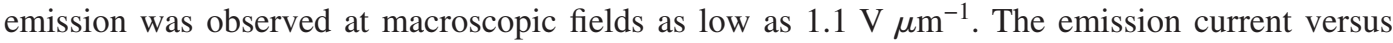
time plot shows significant potential for future field emission applications. () 2006 American Institute of Physics. [DOI: 10.1063/1.2178247]
\end{abstract}

Diamond thin films are a class of carbon materials that have been used for electron field emission devices since 1991. ${ }^{1}$ Its electron emission properties depend on several factors, such as the work function, grain boundaries, ${ }^{2}$ and small protuberances. ${ }^{3-7}$ For example, it has been shown that the boundaries between grains, sites rich in $s p^{2}$ hybridization, emit electrons more easily than the diamond facets of boron-doped diamond (BDD) polycrystalline films. ${ }^{7}$ Interestingly, despite boron being an acceptor state in diamond it appears to enhance the electron emission properties of the material. We believe this is based on the substrate playing an important role in the emission process of diamond films. In fact, it has been observed that better emission results from chemical vapor deposited diamond films grown on $\mathrm{SnO}_{2}$ instead of silicon, suggesting that the electron supply layer or substrates plays an active role in the emission process. ${ }^{8}$ However, despite the well-known attractive properties of diamond (negative electron affinity, thermal, mechanical, and chemical inertness), there are still several problems in the production of field emission devices based on this material associated with obtaining high current densities from emitters, obtaining uniform microstructures, etc., which affect long-term stability. ${ }^{9}$ In addition, diamond films, as well as other carbon materials which have been used for emission, are subject to thermal effects at the interface between the substrate and the carbon film. The presence of heating at the interface of the film and substrate generates different mechanical stresses on the film. This will increase the resistance and/or the voltage barrier needed to maintain electron emission at low voltages for an extended periods of time.

In order to circumvent the above problem, we present here an approach based on a combination of carbon nanotubes (CNTs) and BDD films mounted on a carbon felt to obtain reproducible electron emission at low fields. BDD

\footnotetext{
a)Author to whom correspondence should be addressed; electronic mail: rosolen@ffclrp.usp.br


Campos-12245-970, Brazil.

${ }^{c}$ Present address: Institute of Materials Research and Engineering, 3 Research Link, Singapore 117602.

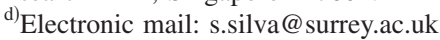

mounts on a carbon felt have previously been explored for electrochemical applications. ${ }^{10,11}$ CNTs grown on BDD films, as described in this letter, is a further interesting technique to increase its potential uses.

The felt substrate was obtained from carbonization of polyacrinitrile at $2000{ }^{\circ} \mathrm{C}$. The obtained carbon fibers have a diameter of around $10 \mu \mathrm{m}$ and resistivity equal to $0.96 \Omega \mathrm{cm}^{-1}$ (determined by the four-probe method). BDD films with acceptor concentrations of about $1.5 \times 10^{21} \mathrm{~cm}^{-3}$ were then grown by the hot filament chemical vapor deposition (HFCVD) method as described in literature. ${ }^{9,10}$ The Raman spectra of polycrystalline BDD films studied in the present letter are identical to those found in Ref. 10, which shows diamond grains with only $s p^{3}$ hybridization. The CNTs were grown on each BDD/carbon fiber using methanol vapor. The catalysts were a mixture of cobalt and manganese. These samples were dispersed on BDD/carbon fiber by immersion of the $\mathrm{BDD} / \mathrm{felt}$ substrate in an ethanol solution

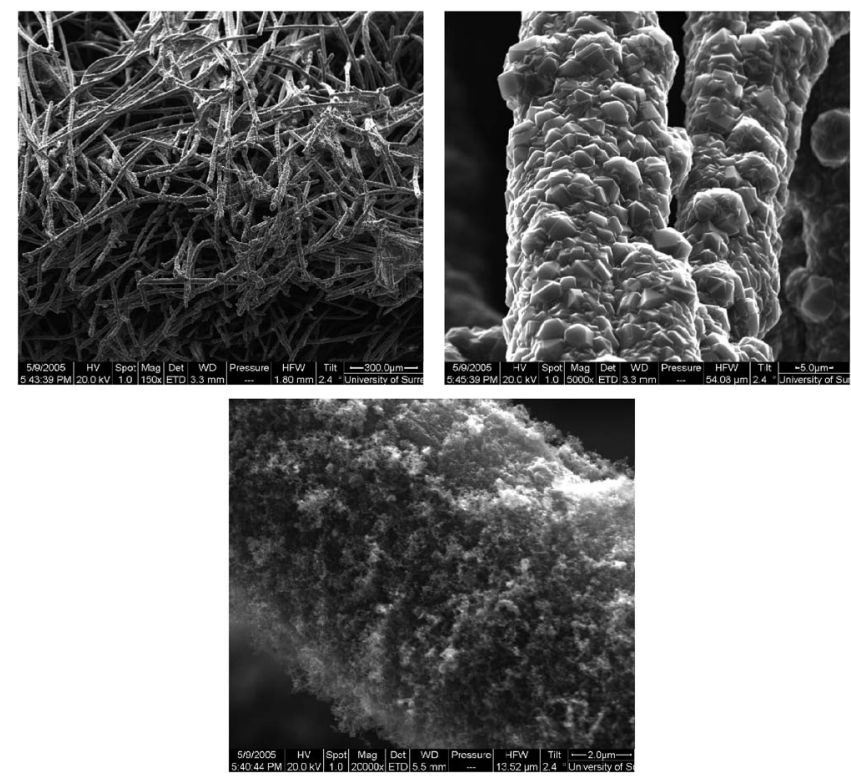

FIG. 1. Typical SEM micrographs of the composite CNT/BDD/felt electrode and single diamond/fiber and single CNT/BDD/fiber. 

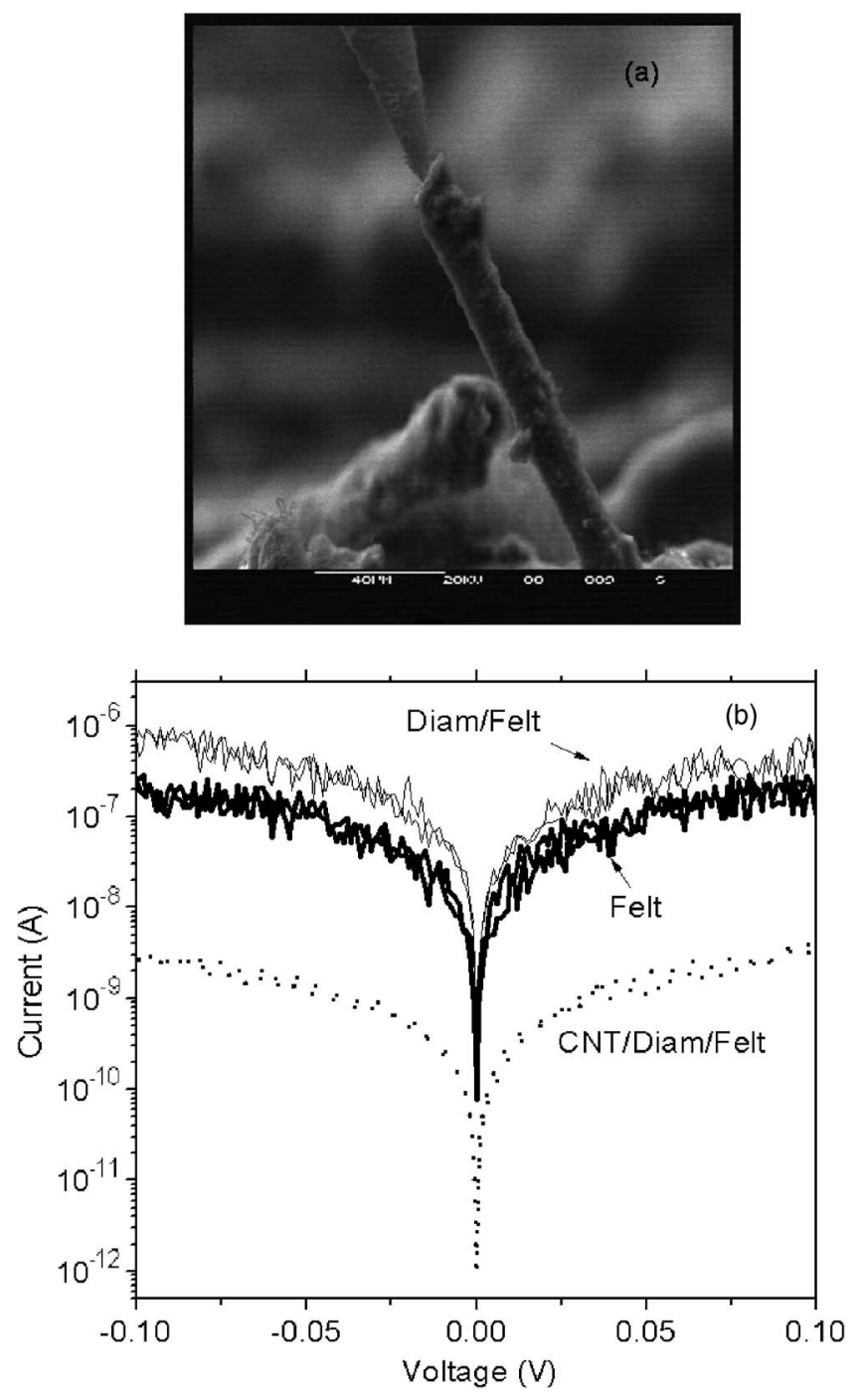

FIG. 2. Compound fibers of electrode in contact with a tungsten probe inside a SEM microscope (A) and its variation of current versus voltage (B).

containing 5 wt. $\%$ of cobalt and manganese acetate and then were left at $80{ }^{\circ} \mathrm{C}$ under ambient conditions. The temperature and time of CNT growth was $650{ }^{\circ} \mathrm{C}$ and $20 \mathrm{~min}$, respectively. $\mathrm{N}_{2}$ was used as the carrier gas for methanol vapor at a constant flow rate of $0.2 \mathrm{ml} \mathrm{min}^{-1}$.

Figure 1 shows scanning electron microscopy (SEM) images from the CNT/BDD/felt electrodes, the polycrystalline BDD film, and the CNT grown on BDD film on a single fiber. The BDD film covered the whole fiber in the felt and the CNT was then grown over the surface of the BDD film. The micrographs show that the CNT growth occurs at the boundary between the grains as well as on the face of each BDD crystal. This suggests that the BDD films (or substrate) should not have a major influence on the type of CNT growth resulting from the catalytic decomposition of methanol.

Indeed, the catalytic particles were observed at the tip of the tubes. If we consider previous studies on the growth of $\mathrm{CNT}$ on zeolite and $\mathrm{NaCl}$, with different catalyst using similar CNT growth conditions as those employed in this study, the CNTs on BDD films should produce a structure similar to bamboo or cup stacked in nature. In our studies, we found multiwalled nanotubes which have a correlation between the structure of the CNT and catalyst particle dimension. The Downloaded 30 Mar 2009 to 131.227 .178 .132 . Redistribution subject

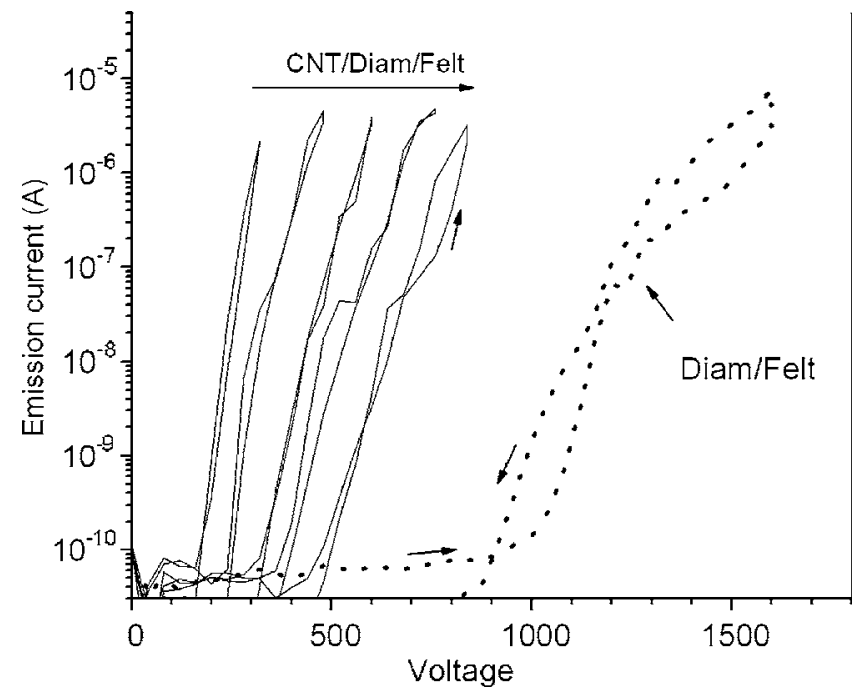

FIG. 3. Emission current vs the applied voltage of the CNT/BDD/felt for several gap distances at $100 \mu \mathrm{m}, 200 \mu \mathrm{m}, 300 \mu \mathrm{m}, 400 \mu \mathrm{m}, 500 \mu \mathrm{m}$, and emission curve of BDD/felt at $500 \mu \mathrm{m}$ gap (dashed line). The curves were collected beginning at $100 \mu \mathrm{m}$ and the distance was increased.

dimension of the catalytic particles at the boundary of diamond grains and face were different. Thus, the CNT on BDD films may be a mixture of bamboolike, cup stacked, or simply disordered multiwalled CNTs.

Figure 2 shows the variation of current versus voltage $(I-V)$ curves for the compound fibers of electrodes in contact with a tungsten probe inside an SEM microscope. In this setup, the felt was grounded using a silver epoxy and the current measured was not associated with a single fiber. Besides this, the curves have the same profile and all electrodes show high electronic conductivity. This allows one to infer that adherence of the BDD to the felt is very good. It also points to a well-adhered CNT deposit on BDD films, i.e., the resistance at the several interfaces of our compound is very low. As a consequence, the Joule heating at these interfaces is expected to be low.

Typical field emission $I-V$ curves for the BBD/felt and CNT/BDD/felt electrodes are shown in Fig. 3. All felt electrode $I-V$ curves were collected using a stainless-steel sphere anode ( $5 \mathrm{~mm}$ diameter), and the composite carbon and felt electrode samples were of dimensions $0.8 \times 0.8 \mathrm{~cm}^{2}$ with a thickness of $0.5 \mathrm{~cm}$. The vacuum chamber was evacuated to about $2 \times 10^{-6}$ Torr prior to electron emission testing, and the electrical contact of the CNT-felt was conducted using a thin layer of silver epoxy. The gap between the sphere and CNT-felt was control by an automated translator with a stepper motor. The BDD/felt is capable of emitting $1 \mathrm{nA}$ at a macroscopic field of $2.2 \mathrm{~V} \mu \mathrm{m}^{-1}$, while the CNT/BDD/felt

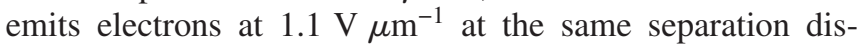
tance from the anode. The turn-on fields for current at $10 \mu \mathrm{A}$

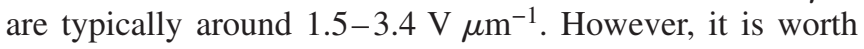
noting that the effective geometric area used in the emission of the felt is very complex and difficult to determine for each fiber taking part in the electron emission process.

Figure 3 also shows a small hysteresis effect on the $I-V$ curves for the composite electrodes, i.e., the difference in the applied fields between the upward and downward voltage cycles appear mainly at an $I-V$ measurement distance of $500 \mu \mathrm{m}$. The results suggest that this hysteresis is associated with geometric factors since it was not observed at lower gap to AIP license or copyright; see http://apl.aip.org/apl/copyright.jsp 


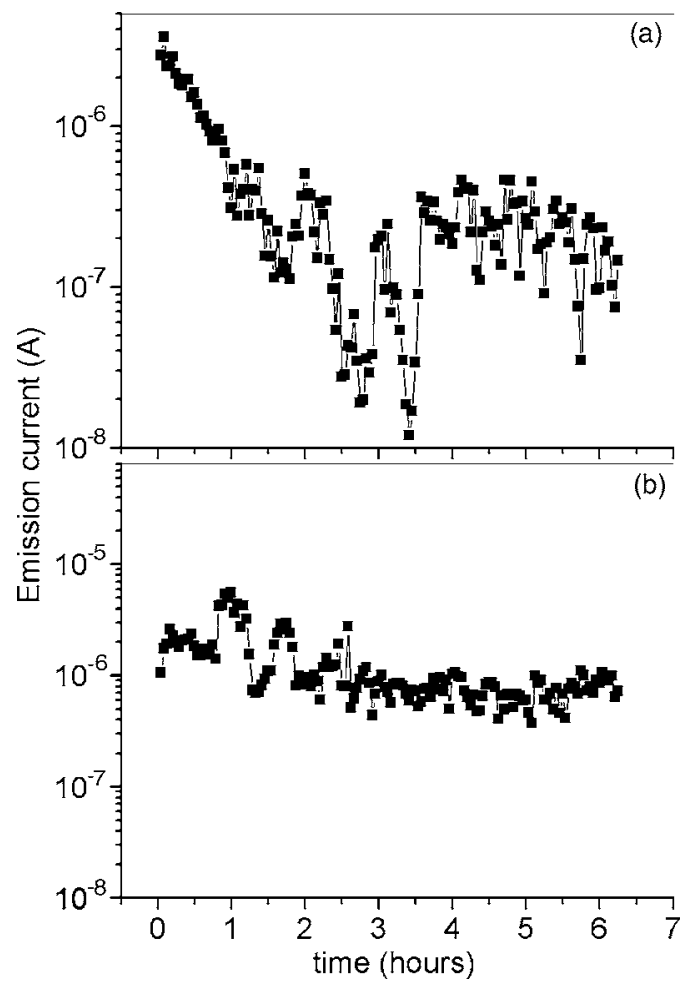

FIG. 4. Variation of emission as function of time current of CNT/BDD/felt electrode: (a) As obtained at $2.1 \mathrm{~V} / \mu \mathrm{m}$ and (b) at $4 \mathrm{~V} / \mu \mathrm{m}$ after (a) run. The voltage was applied without previous voltage scan on electrode.

distances. The presence of amorphous carbon or impurities are often said to be responsible for this hysteresis. Both $I-V$ curves increase monotonically with voltage, with the saturation occurring first for the $\mathrm{BDD} /$ felt electrode. For the CNT/ $\mathrm{BBD} / \mathrm{felt}$, the saturation seems to occur at higher fields than the $\mathrm{BDD} / \mathrm{felt}$. This result suggests that depending on the applied field, the emission of the CNT/BBD/felt may be selectively chosen. At shorter gap separation distances, the emission should be dominated by all forms of carbons of the composite electrode, while at larger separations the emission of the electrode should be predominantly influenced by the CNTs due to the higher-field enhancement factors of these structures. The CNT/BBD/felt appears thus very efficient for emission. This combination was able to provide a variation in the $I-V$ curves that is similar to that observed for singlewall CNTs, where the $I-V$ curves are found to have a sharp rise in emission after the threshold field $E_{\mathrm{th}}{ }^{6,11}$

Finally, Fig. 4 shows the behavior of emission current versus time for $\mathrm{CNT} / \mathrm{BDD} / \mathrm{felt}$ at two different fields. The voltage was applied without any previous voltage scans on the electrodes. The CNT/BDD/felt shows small fluctuations during the first $3 \mathrm{~h}$ of the emission, and then stabilized at

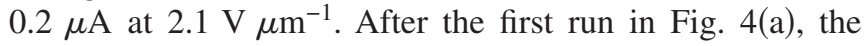
sample was removed from the chamber and then subject to emission tests after long-term exposure to air for a day at room atmosphere. For a field of $4 \mathrm{~V} \mu \mathrm{m}^{-1}$, the electrode emitted about $2 \mu \mathrm{A}$ with good stability. The absence of fluctuations in Fig. 4(b) suggests that this observed variation is associated with some kind of degradation of the CNTs and/or formation of amorphous carbon.

In conclusion, this letter shows the CNT/BDD/felt electrode to be an excellent field emission electrode. This composite electrode has good mechanical properties and can be subject to chemical treatments without significant lost of CNTs. The CNT/BDD/felt is able to emit electrons at voltages in the range of 100-1500 microns at threshold fields that appear smaller than the most of flat multiwall or singlewall CNT electrodes prepared at the present time. This excellent performance is associated with several factors. The first is due the capacity of the felt substrate to mount large amounts of BDD films and CNTs on the emission area. Another important factor is the low resistance of the electrode. Poor electrical contact resulting from low adherence of CNTs or diamond to substrates generally results in a Joule effect that will provoke degradation or failure of emission. In this new approach, the resistance between the CNT-carbon is smaller than CNT-Si or CNT-metal. Third, is the combination of materials that are capable of emitting together and/or to act as a channel for emission of electrons, i.e., BDD film for carbon fiber and CNT for the BDD film. We believe that the carbon compound presented in this letter opens the door for the development of alternative sources for generation of emission devices.

Some authors (J.M.R, S.T., and M.M.) thank CAPES (BEX 0278/04-1), CNPq, and FAPESP. One of the authors (J.M.R.) thanks the University of Surrey for hospitality during his sabbatical leaving. The University of Surrey acknowledges the support from the EPSRC Portfolio Partnership project.

${ }^{1}$ B. C. Djubua and N. N. Chubum, IEEE Trans. Electron Devices 38, 2314 (1991).

${ }^{2}$ S. R. P. Silva, G. A. J. Amaratunga, and K. J. Okano, J. Vac. Sci. Technol. B 557, 17 (1999).

${ }^{3}$ A. Wisitsora-at, W. P. Kang, J. L. Davidson, Q. Li, J. F. Xu, and D. V. Kerns, Appl. Surf. Sci. 280, 146 (1999).

${ }^{4}$ Y. H. Chen, C. Ti, and I. N. Lin, Appl. Surf. Sci. 142, 516 (1999).

${ }^{5}$ Y. Ando, Y. Nishibayashi, H. Furuta, K. Kobashi, T. Hirao, and K. Oura, Diamond Relat. Mater. 12, 1681 (2003).

${ }^{6}$ O. M. Küttel, O. Gröning, Ch. Emmenegge, L. Nilsson, L. Maillard Diederich, and L. Schlapbach, Carbon 745, 37 (1999).

${ }^{7}$ S. Y. Chen, M. Y. Lee, C. S. Chen, and J. T. Lue, Phys. Lett. A 436, A313 (2003).

${ }^{8}$ P. M. Koinkar, R. J. Mahajan, P. P. Patil, and M. A. More, Thin Solid Films 275, 474 (2005).

${ }^{9}$ E. C. Almeida, N. G. Ferreira, and J. M. Rosolen, Diamond Relat. Mater. 14, 1673 (2005)

${ }^{10}$ E. C. Almeida, A. V. Diniz, J. M. Rosolen, and N. G. Ferreira, Diamond Relat. Mater. 14, 679 (2005).

${ }^{11}$ J. M. Bonard, M. Croci, C. Klinke, R. Kurt, O. Noury, and N. Weiss, Carbon 40, 1715 (2002). 